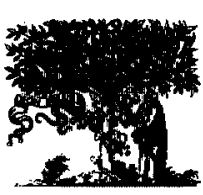

\title{
Characterization of the benzene molecule adsorbed in USY zeolite using double quantum filtered NMR spectral analysis
}

\author{
Yu-Huei Chen, Wen-Tsung Chang, Pang-Chih Jiang, Lian-Pin Hwang * \\ Department of Chemistry, National Taiwan University and Institute of Atomic and Molecular Sciences, Academia Sinica. \\ Taipei, Taiwan
}

Received 25 August 1997; received in revised form 22 December 1997; accepted 22 December 1997

\begin{abstract}
The investigations of deuteron NMR relaxation including $T_{1}$, linewidths and double quantum filtered (DQF) spectral analyses are examined preliminary to probing the dynamics and exchange process of low-loading benzent molecules adsorbed into ultra-stable Y-type (USY) zeolite. DQF NMR is a sensitive method for the determination of the residual quadrupolar interaction resulting from the local order. The usual observed deuteron spectra of benzene- $d_{6}$ in USY retain the isotropic peak with no solid pattern over a broad temperature range. However, when observed in DQF spectra, an additional central line appears. It is evidence of the benzene in isotropic motion exchanging with the benzene situated at adsorption sites with non-vanishing quadrupolar interaction. We modified the cone model to describe the in-plane rotation around the hexad axis and the restricted wobbling motion of the benzene ring on the surface of the interaction site. The population profile of adsorbate thus determined provides useful knowledge of the site distribution in supercages of USY zeolite. Comparison with the result of $\mathrm{NaY}$ in termb of site distribution is also discussed briefly. C 1998 Elsevier Science B.V. All rights reserved.
\end{abstract}

Keywords: USY zeolite; Benzene adsorption; Residual interactions; Quadrupolar relaxation; Double-quantum filtered NMR

\section{Introduction}

There has been great interest in the study of the adsorption process in zeolites by using NMR relaxation techniques $[1,2]$. When observed in DQF spectra adsorbed benzene molecule, an additional central line appears. It is evidence of the benzene in isotropic motion exchanging with the benzene situated at adsorption sites with nonvanishing quadrupolar interaction. For the quad-

\footnotetext{
* Corresponding author. Fax: + 886-2-3636359:
}

e-mail: nmra $(a)$ po.iams.sinica.edu.tw rupolar system of adsorbate molecules, the observed spectra give Lorentzian lines over a broad temperature range. Considering this fact, jump motions among the various adsorption sites may average out the residual interaction on adsorption $[3,4]$. However, from a physical point of view, it seems unreasonable to assume isotropic motion of the adsorbate molecule if it remains attached to the adsorption site for a significant time. We apply the theory of double quantum filtered (DQF) NMR spectroscopy as a diagnostic tool to determine the effects of the residual quadrupolar interaction resulting from the local order [5]. For 
$I=1$ spin system, the double quantum coherence vanishes in the isotropic medium, and hence observation of the $D Q F$ spectra indicates the presence of the anisotropy motion of the adsorbed molecules $[5,6]^{1}$.

In this work, we report the use of deuteron NMR relaxation studies to probe for the dynamic exchange process of benzene molecules adsorbed in USY type zeolite. It is believed that the benzene molecule interacts specifically with the adsorption sites available in the supercages of the zeolite. Therefore, low loading of zeolite with benzene is suitable as a candidate for such study. When observed in DQF spectra, an additional central line appears. It is evidence of the benzene in isotropic motion exchanging with the benzene situated at adsorption sites with non-vanishing quadrupolar interaction. In addition to the usual relaxation studies, we measurcd DQF NMR with different $D Q$ creation periods for the relaxation analyses of exchange process. The motional parameters and the characteristic adsorbate distribution pertinent to the restricted dynamic model provide useful information on the adsorptive properties of the zeolite system.

\section{Model}

\subsection{Two-site exchange model with residual quadrupolar interaction}

The adsorption interaction of per-deuterated benzene in zeolite may be in turn related to a residual quadrupolar interaction $\omega_{\mathrm{q}}$ of deuterium nuclei due to local order. The Hamiltonian of this interaction is given by [5]:

$H=\omega_{\mathrm{q}, \theta}\left[I_{z}^{2}-I(I+1)\right]$

where $\omega_{\mathrm{q} . \theta} \equiv \omega_{\mathrm{q}}\left(3 \cos ^{2} \theta-1\right) / 2$ is the residual quadrupolar interaction in the laboratory frame, and $I_{z}$ is the $z$ component of the spin operator with $I=1$ for deuterium nuclei. $\theta$ is the angle between the local symmetry axis of the quadrupolar inter-

\footnotetext{
${ }^{1}$ There is a misprint in Eq. (32) of ref. [6]. Instead of $5 \sqrt{2} / 7$ as printed, the coefficient of $\left\langle D_{00}^{(2)}\right\rangle$ in $J_{2}\left(2 \omega_{\mathrm{r}}\right)$ should be corrected as $10 / 7$.
}

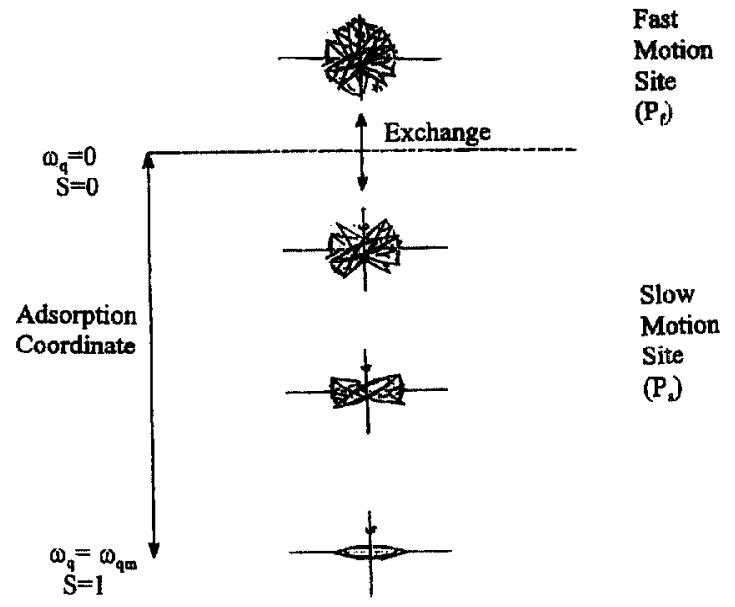

Fig. 1. Schematic representation of benzene adsorption with exchange between the fast ( $f$ ) and slow (s) sites in zeolite. For $s$ sites, an adsorption coordinate is assumed to represent the various strengths of residual adsorption interaction, which are in turn related to the residual quadrupolar interactions and also to the order parameter $(S)$ which describes the extent of restricted wobbling motion of adsorbed benzene.

action tensor and the Zeeman field. A model with two-site exchange between a fast motion site, denoted by f, and a slow motion site, denoted by $\mathrm{s}$, is assumed (see Fig. 1). The $\mathrm{s}$ (or $\mathrm{f}$ ) site accounts for the adsorption (or non-adsorption) state of benzene in USY. An isotropic distribution of $\theta$ is applied to approximate the situation of the random orientational distribution of zeolite crystals in the powder sample.

DQF spectra are conventionally measured using the pulse sequence [5] $\pi / 2-\tau-\pi / 2-\delta-\pi / 2-t$ (Acq.). The effect of this pulse sequence for spin-I nuclei in a heterogeneous system is as follows. The first $\pi / 2$ pulse converts the equilibrium density matrix given by the irreducible tensor $T_{10}$ into the transverse component $T_{11}$ [6]. During the DQ creation period $\tau$, residual quadrupolar interaction transfers $T_{11}$ to $T_{21}$. The second $\pi / 2$ pulse transfers $T_{21}$ to $T_{22}$ and by suitable phase cycling the contribution of the DQ coherence $T_{22}$ is selected ${ }^{2}$.

\footnotetext{
${ }^{2}$ The irreducible tensorial components are defined as $T_{10} \equiv I_{z} / \sqrt{2}, \quad T_{11} \equiv I_{+} / 2, T_{21} \equiv\left(I_{z} I_{+}+I_{+} I_{z}\right)$ and $T_{22} \equiv I_{+} I_{+} / 2$. These expressions correspond to $\hat{Q}_{3}, \dot{Q}_{2}, \dot{Q}_{6}$ and $\dot{Q}_{5}$, espectively, defined in ref. [6].
} 
The last $\pi / 2$ pulse transfers $T_{22}$ into $T_{21}$ which is then converted into observable $T_{11}$ by residual quadrupolar interaction in the acquisition period. In the pulse sequence $\delta=10 \mu \mathrm{s}$ is used to retain the $T_{22}$ coherence after the DQF phase cycling. Hence, in the present relaxation study the evolution of related transverse components $T_{11}$ and $T_{21}$ at sites $\mathrm{f}$ and $\mathrm{s}$, expressed by the superscripts, is needed in the calculation of DQ coherence and is observable by following the rate equation [7]

$$
\frac{\mathrm{d}}{\mathrm{d}}\left[\begin{array}{c}
T_{11}^{\mathrm{f}} \\
T_{21}^{\mathrm{f}} \\
T_{11}^{\mathrm{s}} \\
T_{21}^{\mathrm{s}}
\end{array}\right]=\left[\begin{array}{cccc}
-R_{11}^{\mathrm{f}}-k_{\mathrm{ss}} & 0 & k_{\mathrm{ss}} & \\
0 & -R_{21}^{\mathrm{f}}-k_{\mathrm{fs}} & 0 & 0 \\
k_{\mathrm{fs}} & 0 & -R_{11}^{\mathrm{s}}-k_{\mathrm{fs}} & -\mathrm{i} \omega_{\mathrm{q}, t} \\
0 & k_{\mathrm{fs}} & -i \omega_{\mathrm{q}, a} & -R_{21}^{\mathrm{s}}-k_{\mathrm{fs}}
\end{array}\right]\left[\begin{array}{c}
T_{11}^{\mathrm{f}} \\
T_{21}^{\mathrm{f}} \\
T_{11}^{\mathrm{s}} \\
T_{21}^{\mathrm{s}}
\end{array}\right]
$$

where the residual quadrupolar interaction exists in s sites only ${ }^{3}, k_{\mathrm{fs}}\left(k_{\mathrm{sf}}\right)$ is the microscopic rate constant for transfer from site $f(s)$ to site $s$ (f). In addition, the detailed balancing of the exchange process demands that $P_{\mathrm{p}} k_{\mathrm{rs}}=P_{\mathrm{s}} k_{\mathrm{s}}$, where we define $P_{\mathrm{f}}$ and $P_{\mathrm{s}}$ as the total populations in site $f$ and site $s$, respectively. In Eq. (2), the relaxation rate constants for single and double quantum coherences (expressed by the first subscript of $R$ ) with different sites $\alpha(\alpha=\mathrm{s}$ or f) are given by $R_{11}^{\alpha}=C^{\alpha}\left(3 J_{0}^{\alpha}+5 J_{1}^{\alpha}+2 J_{2}^{\alpha}\right)$ and $R_{21}^{\alpha}=$ $C^{\alpha}\left(3 J_{0}^{\alpha}+J_{1}^{\alpha}+2 J_{2}^{\alpha}\right) \quad$ where $\quad C^{\prime}=C^{s} \equiv(3 / 160) \chi^{2}$ with the same quadrupolar coupling constants $\chi \equiv e^{2} q Q / h$ assumed for both sites [6]. The contribution of the asymmetry parameter $(\eta-0.041)$ of the electric field gradient eq to the coupling constant is neglected [8]. $J_{n}^{\mathrm{f}}$ and $J_{n}^{\mathrm{s}}$ are the spectral density functions defined below for reorientational relaxation of benzene molecules in $f$ and $s$ sites, respectively. Starting from converting the equilibrium magnetization into transverse

\footnotetext{
${ }^{3}$ The simulation of spectra from the Liouville equation represented by the density operator with exchange process is described. As shown in ref. [6], the representation ofthe Liouville operator on the basis of $\left[T_{11}^{\mathrm{s}}, T_{21}^{\mathrm{s}}\right]$ corresponding to the residual quadrupolar interaction [cf. Eq. (1)] is given by $\left[\begin{array}{cc}11 & i \omega_{q, \theta} \\ i\left(\omega \omega_{4, \theta}\right. & 0\end{array}\right]$. This result with relaxation and exchange formulation is incorporated in Eq. (2).
}

magnetization, we have $T_{11}^{\mathrm{f}}(0)=P_{\mathrm{f}} T_{11}(0)$ and $T_{11}^{\mathrm{s}}(0)=P_{\mathrm{s}} T_{11}(0)$. Without losing generality, $T_{11}(0)$ may correlate with the equilibrium magnetization $M_{0} \quad\left[M_{0} \equiv \sqrt{2} T_{10}(0)\right]$ as $T_{11}(0)=M_{0} / 2$ after applying an initial $\pi / 2$ pulse. The $D Q F$ spectra are simulated using the above formulation for $T_{21}^{\mathrm{f}}$ and $T_{21}^{\mathrm{s}}$ evolving throughout the DQ creation period $\tau$ and the subsequent results $T_{21}^{\mathrm{f}}(\tau)$ and $T_{21}^{\mathrm{s}}(\tau)$ are used as the initial condition for evolution of observable $\left[T_{11}^{\mathrm{f}}(t)+T_{11}^{\mathrm{s}}(t)\right]$ in the acquisition period. This equation is also used to calculate $\left[T_{11}^{\mathrm{f}}(t)+T_{11}^{\mathrm{s}}(t)\right]$ for single quantum spectra. Analogous simulations of the spectra were described elsewhere [7]. In the calculation of $T_{1}$, the time evolution of the longitudinal components follows the rate equation [6]

$\frac{\mathrm{d}}{\mathrm{d} t}\left[\begin{array}{c}T_{10}^{\mathrm{f}} \\ T_{10}^{\mathrm{s}}\end{array}\right]=\left[\begin{array}{cc}-R_{10}^{\mathrm{f}}-k_{\mathrm{fs}} & k_{\mathrm{sf}} \\ k_{\mathrm{fs}} & -R_{10}^{\mathrm{s}}-k_{\mathrm{sf}}\end{array}\right]\left[\begin{array}{c}T_{10}^{\mathrm{f}} \\ T_{10}^{\mathrm{s}}\end{array}\right]$

where $R_{10}^{\mathrm{f}}$ and $R_{10}^{\mathrm{s}}$ are the longitudinal relaxation rates for site $f$ and site $s$, respectively, as defined by $R_{10}^{\alpha}=2 C^{\alpha}\left(J_{1}^{\alpha}+4 J_{2}^{\alpha}\right)$.

\subsection{Residual quadrupolar interaction in adsorptive process}

The position with maximum $\omega_{\mathrm{q}}$, denoted by $\left(\omega_{\text {qm }}\right.$, may he defined as the benzene molecule adsorbed in the adsorption site with maximum strength of interaction. Moreover, the motion associated with $\omega_{\mathrm{qm}}$ involves in-plane motion around the hexad axis only, while there is no wobbling motion of the ring normal. Thus, from both the experimental evidence and the symmetry arguments resulting from the fast internal motion around the hexad axis. the quadrupolar interaction of per. deuterated benzene molecules in this case reduces to half of the value obtained for rigid stationary benzene [3]. Hence we take $\omega_{\mathrm{qm}}=(1 / 2) \omega_{\mathrm{qo}}$ where $\omega_{\mathrm{q} 0}=(3 / 4) \chi$. Experimentally, $\omega_{\mathrm{qm}}$ may be determined from the edge splitting of the solid pattern of adsorbed benzene and we obtain $\omega_{\mathrm{qm}} / 2 \pi=67$ $\mathrm{kHz}$ at $130 \mathrm{~K}$.

It should be noted that there exists a difference: in adsorbate population along the adsorption coor. 
dinate as a consequence of exchange and migration of adsorbate among various sites in the supercage. A probability distribution may be represented suitably in an exponential form $f\left(\omega_{\mathrm{q}}\right)=$ $N \exp \left(-\omega_{\mathrm{q}} / \omega_{\mathrm{qc}}\right)$ where $\omega_{\mathrm{qc}}$ characterizes a correlation frequency for resident sites in $\omega_{\mathrm{q}}$ space. Beyond this value the population of adsorbate molecules decreases significantly and $N$ is a normalization factor for $f\left(\omega_{\mathrm{q}}\right)$. It should be noted that all the calculated results are averaged over the adsorbate distribution.

\subsection{Spectral density function for benzene in fast motion sites}

In order to match the central peak widths of the DQF spectra, the motional narrowing conditions are required for $\tau_{\mathrm{f}}$ which is defined as the tumbling correlation times of benzene in $f$ site. Hence we have $J_{n}^{f} \approx 2 \tau_{\mathrm{f}}$. The simulated results are insensitive to these two values as long as their magnitudes are kept of the order of less than $10^{-10} \mathrm{~s}$

\subsection{Spectral density function for benzene in slow motion sites}

The spectral density functions for site $\mathrm{s}$ with consideration of residual quadrupolar interaction are defined by [6]:

$$
\begin{aligned}
& J_{0}^{\mathrm{s}} \equiv J_{0}^{\mathrm{s}}\left[1+(10 / 7)\left\langle D_{00}^{(2)}\right\rangle+(18 / 7)\left\langle D_{00}^{(4)}\right\rangle-\left\langle D_{00}^{(2)}\right\rangle^{2}\right] \\
& J_{1}^{\mathrm{s}} \equiv J_{1}^{\mathrm{s}}\left[1+(5 / 7)\left\langle D_{00}^{(2)}\right\rangle-(12 / 7)\left\langle D_{00}^{(4)}\right\rangle\right] \\
& J_{2}^{\mathrm{s}} \equiv J_{2}^{\mathrm{s}}\left[1-(10 / 7)\left\langle D_{00}^{(2)}+(3 / 7)\left\langle D_{00}^{(4)}\right\rangle\right]\right.
\end{aligned}
$$

where $D_{00}^{(n)}$ are the Wigner rotation matrix elements with the orientation relative to the laboratory frame, and $\langle\cdots\rangle$ means taking the average over the coordinates of in-plane and wobbling motions. The cone model of Brainard and Szabo [9] is invoked, but with minor modification to consider the residual interaction and hence to facilitate the calculation of the spectral density functions $f_{n}^{s}$ of the adsorbed benzene molecule. It is given by $[9,10]^{4}$ :

$$
\begin{aligned}
J_{n}^{s} \equiv & 2\left\{\frac{3}{4} S^{2} \frac{4 D_{\mathrm{i}}}{\left(4 D_{\mathrm{i}}\right)^{2}+\left(n \omega_{0}\right)^{2}}\right. \\
& +\frac{3}{4}\left(1-S^{2}\right) \frac{4 D_{\mathrm{i}}+2 D_{\mathrm{w}} /\left(1-S^{2}\right)}{\left[4 D_{\mathrm{i}}+2 D_{\mathrm{w}} /\left(1-S^{2}\right)\right]+\left(n \omega_{0}\right)^{2}} \\
& \left.+\frac{1}{4}\left(1-S^{2}\right) \frac{6 D_{\mathrm{w}} /\left(1-S^{2}\right)}{\left[6 D_{\mathrm{w}} /\left(1-S^{2}\right)\right]^{2}+\left(n \omega_{0}\right)^{2}}\right\}
\end{aligned}
$$

where $\omega_{0}$ is the Larmor frequency of deuterium nuclei. It is convenient to define the correlation times for the internal rotation and the wobbling motion as $\tau_{\mathrm{i}} \equiv 1 / 4 D_{\mathrm{i}}$ and $\tau_{\mathrm{w}} \equiv 1 /\left(6 D_{\mathrm{w}}\right)$, respectively. The wobbling motion is assumed to be within a cone with the director normal to the adsorption site (see Fig. 1). The distribution of the cone may be expressed by an order parameter $S$ given by $S \equiv\left\langle D_{00}^{\{2\}}\right\rangle_{\mathrm{s}}$ where the average over the wobbling motion is defined in the frame $s$ of an adsorptive site. With this model the second and third terms in Eq. (4) may be readily evaluated as $\left\langle D_{00}^{(2)}\right\rangle=\operatorname{SD}_{00}^{(2)}(\theta)$ and $\left\langle D_{00}^{(4)}\right\rangle=\left\langle D_{00}^{(4)}\right\rangle_{s} D_{00}^{(4)}(\theta)$, where axial symmetry of the adsorption interaction and site distribution are assumed. To evaluate the $\left\langle D_{00}^{(4)}\right\rangle_{\mathrm{s}}$ term in terms of $S$, an isotropic distribution within a cone angle of $\beta$ with the director is invoked. Brainard and Szabo obtained $S=$ $\cos \beta(1+\cos \beta)$. We then yield $\left\langle D_{00}^{(4)}\right\rangle_{\mathrm{s}}=S[(7 / 2)$ $\left.S-7(1+8 S)^{1 / 2}+1\right]$. Hence with this model one may evaluate the spectral density functions for site $s$. Furthermore, it should be noted that $\tau_{\mathrm{i}}$ and $\tau_{\mathrm{w}}$ are used in an averaged sense, i.e. their magnitudes remain the same irrespective of the variation of $S$ along the adsorption coordinates at a given temperature.

\subsection{Order parameter and residual quadrupolar interaction}

Since both the $S$ and $\omega_{\mathrm{q}}$ parameters share the same degree of randomness after averaging by the same wobbling motion, one may relate $S$

\footnotetext{
${ }^{4}$ Comparing Eq. (22) with Eq. (15) in ref. [10], one may derive Eq. (5) of the present work by leaving out the terms related to the overall reorientation motion.
} 
to the residual quadrupolar anisotropy $\omega_{\mathrm{q}}$ by $S=\omega_{\mathrm{q}} / \omega_{\mathrm{qm}}$. The pictorial description of the order parameter and related motions along the adsorption coordinate is depicted in Fig. 1. Furthermore, the adsorbate distribution function may be expressed in terms of $S_{\mathrm{c}}$ with the definition $S_{\mathrm{c}}=\omega_{\mathrm{qc}} / \omega_{\mathrm{qm}}$.

\section{Experimental}

USY zeolite $(\mathrm{Si} / \mathrm{Al}=40)$ and $\mathrm{NaY}$ zeolite $(\mathrm{Si} / \mathrm{Al}=2.5)$ used were supplied and characterized by PQ Zeolites, Holland. The sample in $10 \mathrm{~mm}$ NMR tube containing $\mathrm{C}_{6} \mathrm{D}_{6}$ with an average loading of 0.5 benzene molecule per supercage was carefully prepared and sealed in a vacuum system. The sample was then kept at $353 \mathrm{~K}$ for $12 \mathrm{~h}$ to ensure a homogeneous distribution of adsorbate within the zeolite. The ${ }^{2} \mathrm{D} T_{1}$ and linewidth measurements were performed on Bruker MSL-500 and MSL-300 spectrometers operating at $76.78 \mathrm{MHz}$ $(11.75 \mathrm{~T})$ and $46.07 \mathrm{MHz}(7.05 \mathrm{~T})$, respectively. The DQF spectra were displayed at $76.78 \mathrm{MHz}$ only.

\section{Results and discussion}

The experimental and calculated $T_{1}$ and linewidth obtained at two fields are listed in Table 1. Except for the $T_{1}$ data for the USY system at $76.78 \mathrm{MHz}$ and $210 \mathrm{~K}$, the calculated results agree excellently with the experimental ones. Field

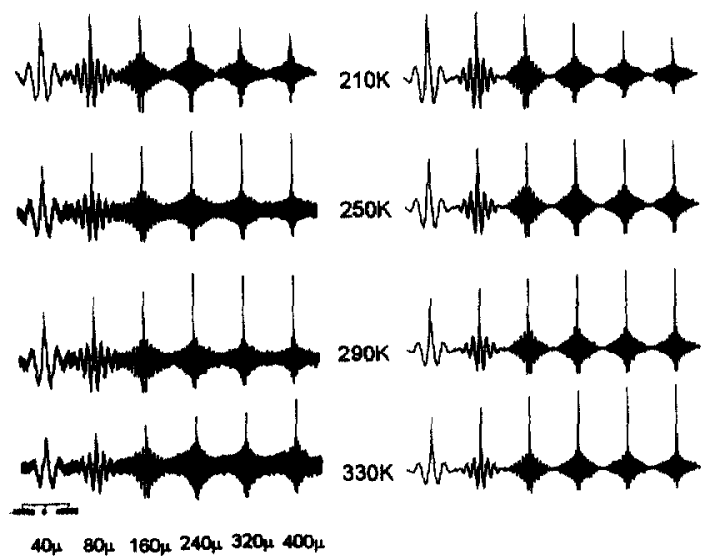

Fig. 2. Experimental (left) and simulated (right) ${ }^{2} \mathrm{D}$ double quantum filtered spectra of a $\mathrm{C}_{6} \mathrm{D}_{6} /$ USY sample at the various temperatures cited. The spectral width of every measurement is $\pm 41667 \mathrm{~Hz}$. The corresponding DQ creation period $\tau$ is 40,80 , $160,240.320$, and $400 \mu$ s, respectively.

dependence is observed in $T_{1}$ data but not observed in linewidths and DQF spectra. One may attribute the dispersion to the non-motional narrowing condition of wobbling motions. Fig. 2 shows the experimental DQF spectra together with their corresponding simulations at various temperatures cited. It is found that within a short creation period ( $\tau \sim 40 \mu \mathrm{s})$ the DQF spectra are sensitive to the variation of $P_{\mathrm{s}}$ and $S_{\mathrm{c}}$ only, but for longer creation periods they are sensitive to the $\tau_{w}$ value as well. The obtained parameters are listed in Table 2. It is believed that the adsorption site of benzene in USY is located at an $\mathrm{OH}$ group in the supercage since the Lewis acidity of hydroxyl

Table 1

Experimental and calculated ${ }^{2} \mathrm{D} T_{1}$ and full linewidth of $\mathrm{C}_{6} \mathrm{D}_{6} / \mathrm{USY}$ sample at half-height measured at $46.07 \mathrm{MHz}$ and $76.78 \mathrm{MHz}$

\begin{tabular}{|c|c|c|c|c|c|c|c|c|}
\hline \multirow[t]{3}{*}{ Temperature ( $\mathbf{K}$ ) } & \multicolumn{4}{|c|}{$T_{1}(\mathrm{~ms})$} & \multicolumn{4}{|c|}{$\Delta v_{1 / 2}(H z)$} \\
\hline & \multicolumn{2}{|c|}{$46.07 \mathrm{MHz}$} & \multicolumn{2}{|c|}{$76.78 \mathrm{MHz}$} & \multicolumn{2}{|c|}{$46.07 \mathrm{MHz}$} & \multicolumn{2}{|c|}{$76.78 \mathrm{MHz}$} \\
\hline & Calc. & Exp. & Calc. & Exp. & Calc. & Exp. & Calc. & Exp. \\
\hline 210 & 44 & 39 & 52 & 73 & 1580 & 1690 & 1525 & 1600 \\
\hline 250 & 37 & 33 & 57 & 57 & 596 & 540 & 587 & 571 \\
\hline 290 & 37 & 35 & 61 & 58 & 228 & 230 & 201 & 219 \\
\hline 330 & 45 & 51 & 69 & 76 & 148 & 150 & 136 & 141 \\
\hline
\end{tabular}

$10 \%$ error is expected in experimental $T_{1}$ and linewidths. 
Table 2

The parameters used in the fitting of $T_{1}$, linewidths and DQF spectra of $\mathrm{C}_{6} \mathrm{D}_{6} / \mathrm{USY}$

\begin{tabular}{llllll}
\hline Temperature $(\mathrm{K})$ & $P_{\mathrm{s}}$ & $S_{\mathrm{c}}$ & $\tau_{\mathrm{i}}(\mathrm{ns})$ & $\tau_{\mathrm{w}}(\mathrm{ns})$ & $k_{\mathrm{fs}} \times 10^{-2}\left(k_{\mathrm{sf}} \times 10^{-2}\right)\left(\mathrm{s}{ }^{-1}\right)$ \\
\hline 210 & 0.92 & 1.10 & $0.11 \pm 0.01$ & $70 \pm 5$ & $46.0(4.0)$ \\
250 & 0.80 & 1.39 & $0.08 \pm 0.01$ & $16 \pm 2$ & $23.5(5.9)$ \\
290 & 0.68 & 1.67 & $0.06 \pm 0.02$ & $7.5 \pm 1$ & $8.5(4.0)$ \\
330 & 0.60 & 5.00 & $0.04 \pm 0.03$ & $4.5 \pm 3$ & $4.6(3.1)$ \\
\hline
\end{tabular}

$10 \%$ error is expected in $k_{\mathrm{fs}}$ and $k_{\mathrm{sf}}$ and $5 \%$ error in $P_{\mathrm{s}}$ and $S_{\mathrm{c}}$
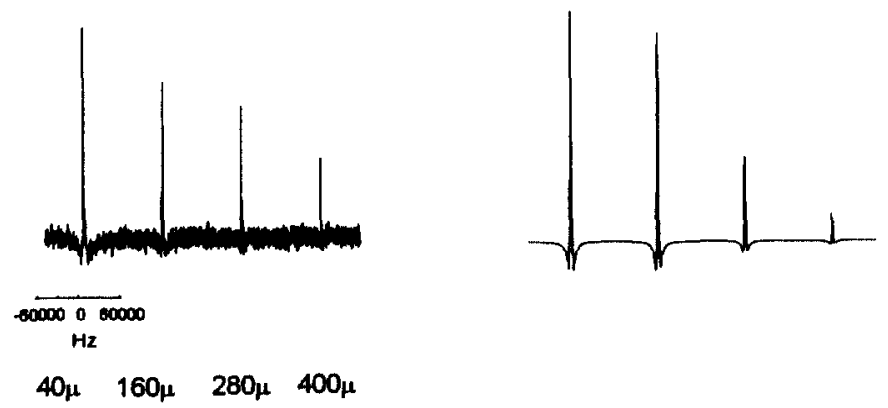

Fig. 3. Experimental (left) and simulated (right) ${ }^{2} \mathrm{D}$ double quantum filtered spectra of $\mathrm{C}_{6} \mathrm{D}_{6} / \mathrm{NaY}$ sample at $310 \mathrm{~K}$ at $76 . \% 8 \mathrm{MHz}$. The spectral width is $\pm 62485 \mathrm{~Hz}$. The corresponding DQ creation period $\tau$ is $40,160,280$, and $400 \mu$, respectively. $T_{1}=72 \pm 7 \mathrm{~ms}$ and $\Delta v_{1 / 2}=3082 \pm 308 \mathrm{~Hz}$ are used in simulation to get the following parameters: $P_{\mathrm{s}}=0.98, \quad S_{\mathrm{c}}=4.48 \times 10^{-3}$. $\tau_{\mathrm{i}}=50 \mathrm{~ns}, \tau_{\mathrm{w}}=180 \mathrm{~ns}, k_{\mathrm{fs}}=1.51 \times 10^{3} \mathrm{~s}^{-1}$, and $k_{\mathrm{sf}}=31 \mathrm{~s}^{-1}$.

hydrogen favours the formation of H-bonding with $\pi$-electrons of the benzene ring [11]. Based on the $\mathrm{Si} / \mathrm{Al}$ ratio of the USY sample, the average number of $\mathrm{OH}$ groups per supercage of our USY sample is calculated to be 0.57 . However, one cannot be sure whether the locations of the hydroxyls are in the supercage or in the sodalite cage. As with the loading of 0.5 benzenes per supercage in our sample, the adsorption site is oversaturated with benzene. Therefore, the obtained $P_{\mathrm{f}}$ value and the narrow linewidths of the central component of $D Q F$ spectra can be rationalized by the presence of a considerable benzene distribution in the unbounded $f$ state. Owing to a small number of adsorption sites in the supercage, the adsorbate molecules experience an orderly directed quadrupolar interaction or a significant strength of residual $\omega_{\mathrm{q}}$ interaction. Therefore, a large $S_{\mathrm{c}}$ in the adsorbate distribution may be expected and the resulting effect shows the wiggling pattern observed in the DQF spectra. In the case of $C_{6} D_{6} / \mathrm{NaY}$, the tetrahedrally arranged $S_{I I}$ and 12-ring window sites for benzene adsorption [12] may result in a small
$S_{\text {c }}$, and hence a double quantum spectrum without complicated wiggling in the wing is obtained in accordance with simulation as shown in Fig. 3.

The increasing of $k_{\mathrm{sf}}$ and the decreasing of $P_{\mathrm{s}}, \tau_{\mathrm{j}}$ and $\tau_{\mathrm{w}}$ with temperature reflects the fact that benzene becomes much more mobile within USY frameworks. The magnitude of $\tau_{i}$ varies from $4 \times 10^{-11} \mathrm{~s}$ to $1.1 \times 10^{-10} \mathrm{~s}$ in the temperature range $330 \mathrm{~K}$ to $210 \mathrm{~K}$. The correlation times are about two orders slower than the free diffusion values calculated from $\sqrt{I / k T}$ where $I$ is the moment of inertia around the hexad axis of $\mathrm{C}_{6} \mathrm{D}_{6}$. From molecular dynamics calculations in liquid benzene, it is evident that it gives an in-plane correlation time of about 0.1 ps at $312 \mathrm{~K}$; about twice that of any in-plane $C_{2}$ axis [13]. Hence the $\tau_{\mathrm{i}}$ process of adsorbed benzene is significantly hindered as compared to the motions either in liquid benzene or in gas state. It is also found that the $\tau_{\mathrm{j}}$ process in USY yields a lower activation energy than $\tau_{\mathrm{w}}$ does $\left(\sim 5 \mathrm{~kJ} \mathrm{~mol}^{-1}\right.$ vs. $13 \mathrm{~kJ} \mathrm{~mol}^{-1}$ ). We attribute the small activation energy to the effect of decoupling from another 
motion mode and also to the optimal environment for in-plane rotation of benzene molecules. Aiso, it is interesting to know that in the present case the wotbiing motion is more than two orders siower than the in-piane motion. The effect of interacting forces, which appears in the surrounding adsorption site, may account for the slowing down of the wobbling motion.

In accordance with our analysis of DQF spectra at $330 \mathrm{~K}$, the results are not as satisfactory as comparative studies al lower temperatures. The effect may correlate with the intercage diffusion of the adsorbate occurring in USY and also the intracage motion with increasing temperature [14]. The reverse temperature trend found in $k_{\text {sf }}^{\prime}$ and $S_{c}$ values may be due to the effect of intercage diffusion.

\section{Conclusion}

The 'isotropic' behavior apparent in a single quantum spectrum does not reflect the absence of residual adsorption interactions in the adsorptive process. The traditional methods of analyzing NMR relaxation of adsorbate in zeolite system have not taken the exchange process and the adsorbate distribution into account. The in-plane rotation of adsorbed benzene molecules with low activation energy reflects a small host-guest effect of the isolated adsorption site. The wobbling motion represents a more realistic physical picture of benzene dynamics and the strength of site interaction. Furthermore, results of $S_{\mathrm{c}}$ values may be used to differentiate the nature of adsorption sites in the supercage of different faujasites. Further detaits of the theory and its application to different types of zeolites with yarious adsorbate loadings will be reported elsewhere.

\section{Acknowledgement}

Grateful acknowledgement is made of the valuable discussions with Professor Pao Lian Su and also the support of this work by the National Science Council, ROC.

\section{References}

[1] B. Zibrowius, J. Caro, H. Pfeifer, J. Chem. Soc., Faraday Trans. I 84 (1988) 2347.

[2] D. Barthomeuf, Catal. Rev. 38 (1996) 521 and references cited therein

[3] B. Boddenberg, R. Burneister, Zeolites \& (1988) 488.

[4] B. Boddenberg, B. Beerwerth, J. Phys. Chem. 93 (1989) 1440.

[5] Y. Sharf, U. Eliav, H. Shinar, G. Navon, I. Magn. Reson. B107 (1995) 60 and references cited therein

[6] J.P. Jacobsen, H.K. Bildsoe, K. Schaumburg, J. Magn. Reson. 23 (1976) 153.

[7] W.S. Price, N.H. Ge, L.Z. Hong, L.P. Hwang, J. Am. Chem. Soc. 115 (1993) 1095.

[8] F.S. Millett, B.P. Dailey, J. Chem. Phys. 56 (1972) 3249.

[9] J.R. Brainard. A. Szabo, Biochemistry 20 (1981) 4618

[10] T.C. Wong, P.L. Wang, D.M. Duh, L.P. Hwang, J. Phys Chem. 93 (1989) 1295.

[11] B.L. Su, D. Barthomeuf, Zeolites 13 (1993) 626 .

[12] B.L. Su, J. Chem. Soc., Faraday Trans. 93 (1997) 1449.

[13] P. Lines, S. Engström, B. Jönsson, Chem. Phys. Lett. 115 (1985) 95.

[14] G. Schrimpf, B. Tavitian, D. Espinat, J. Phys. Chem. 99 (1995) 10932 